\title{
Kein Vertrauen in Pertussis-Impfung
}

\author{
Zu einer stringenteren Reimmunisierung erwachsener Patienten gegen Pertussis rät ein Experte \\ in MMW 13/2017, S. 20. Eine Leserin macht auf gravierende Probleme in der Praxis aufmerksam.
}

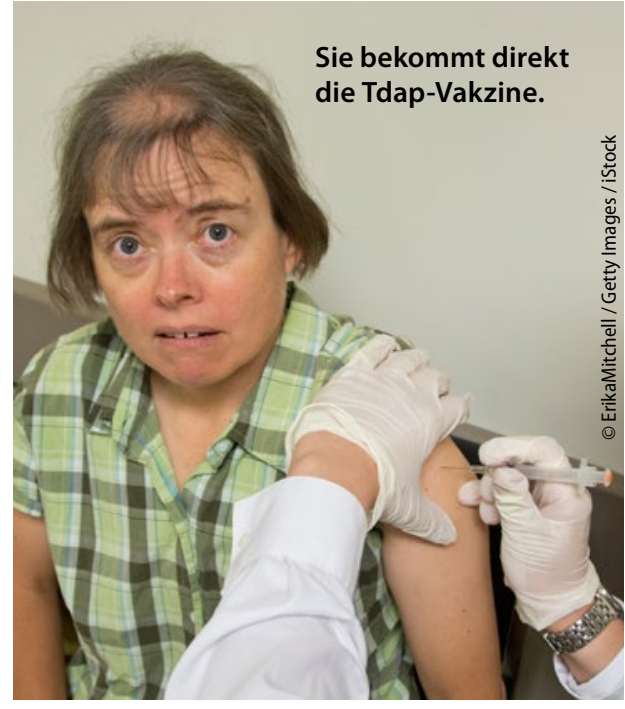

_ Das erste Problem ist die hohe Impfversagerquote. Immer wieder hatte ich in meiner Praxis Fälle von Pertussis trotz Impfung. Dass das ein allgemeines Problem zu sein scheint, erfuhr ich über unser Gesundheitsamt. Dafür scheint sich aber niemand $\mathrm{zu}$ interessieren. Die "UAW-Bögen“ habe ich immer brav an die Pharmafirmen geschickt.

Es nützt nichts, immer wieder auf die Notwendigkeit von Impfungen hinzuweisen, wenn die Impfstoffe nicht hinreichend wirksam sind. Die Impfmotivation von erkrankten Personen wird dadurch auch nicht gerade erhöht.

Das zweite Problem ist organisatorischer Art. Es klingt ganz einfach, bei der anstehenden Tetanus-Auffrischimpfung Pertussis mitzuimpfen. Das könnte man mal einem diensthabenden Arzt in einer Krankenhaus-Ambulanz sagen. Dort wird nämlich nur Tetanus/Diphtherie -Impfstoff verabreicht. Da es leider keinen Pertussis-Monoimpfstoff mehr gibt, ist man nicht mehr flexibel und kann die nächsten Jahre eine Keuchhusten-Prophylaxe schon wieder vergessen.

Und dann gibt es auch noch hin und wieder den guten alten Lieferengpass. Eigentlich war ich immer eine überzeugte Impf-Hausärztin. Aber mein Engagement bekommt zunehmend Risse.

Dr. Aglaia Gietl,

Münchner Str. 43, D-85221 Dachau

\section{Der Quick-Wert gehört endlich beerdigt!}

\section{Die Antikoagulation von älteren Patienten behandelten wird anhand zweier exemplarischer Fälle in MMW 3/2017 ab S. 43. Dabei verwendeten wir sowohl den Quick-Wert als auch die INR, was ein Leser kritisiert.}

_ Ich möchte mit Nachdruck festhalten, dass in der Medizin Quick-Werte im Kontext der Einstellung einer oralen Antikoagulation heute als absolut obsolet angesehen werden sollten. Sowohl die Deutsche Herzstiftung wie auch wiederholt verschiedene Kollegen unserer kardiologischen Fachgesellschaft haben sich in dieser Richtung geäußert.
Deutschland ist nahezu das einzige Land in Europa, in dem Haus- wie auch Fachärzte Patienten mit Vorhofflimmern noch immer nach Quick-Werten einstellen. Selbst in Entlassbriefen, gelegentlich sogar internistischer Abteilungen, sind derartige Empfehlungen noch zu finden. Diese laxe Praxis birgt das Risiko der Über- und Unter-Antikoagulation und trägt maßgeblich dazu bei, dass Deutschland in der Qualität der Einstellung der Antikoagulation mit VitaminK-Antagonisten in Europa lediglich eine Mittelstellung einnimmt!

Prof. Dr. Thomas Klingenheben, Im Mühlenbach 2B, D-53127 Bonn

\section{Antwort von Prof. Heppner}

Wie Sie zu Recht anmerken, sollte der Quick-Wert kein Richtwert mehr im klinisch-praktischen Alltag sein. Da es sich bei den Fallbespielen um Berichte „aus dem wirklichen Leben " handelt und die Anwendung leider immer noch weit verbreitet ist, wollten wir dies nicht unerwähnt lassen. In der Tat wäre es sinnvoll gewesen, dies in der abschließenden Darstellung noch einmal mit Nachdruck $\mathrm{zu}$ betonen. Wir werden dies für die $\mathrm{Zu}$ kunft berücksichtigen.

Prof. Dr. Hans Jürgen Heppner, Helios Klinikum Schwelm, Dr.-Moeller-Str. 15, D-58332 Schwelm 